\title{
Recent Research and Knowledge on Treatment for Hepatitis B
}

\author{
Hye-won Lee, Yongwook Choi, Yong Kwang Park* \\ Division of Viral Disease Research, Center for Infectious Disease Research, Korea National Institute of Health, Chungbuk \\ 28159, Republic of Korea
}

\author{
Corresponding \\ Yong Kwang Park, Ph.D. \\ Division of Viral Disease Research, \\ Center for Infectious Disease Research, \\ Korea National Institute of Health, 187 \\ -ro, Osong-yeup, Cheongju, Chungbuk, \\ 28159, Republic of Korea \\ Phone : +82-43-719-8431 \\ Fax : +82-43-719-8459
}

E-mail : yk1029@korea.kr

Received : September 2, 2020

Revised : September 22, 2020

Accepted : September 23, 2020

No potential conflict of interest relevant to this article was reported.

Copyright (C) 2020 Journal of Bacteriology and Virology

(C) This is an Open Access article distributed under the terms of the Creative Commons Attribution Non-Commercial

License

(http://creativecommons.org/

license/by-nc/3.0/).
Hepatitis B virus (HBV) infection is a major public health problem, with some 250 million people currently at high risk of developing chronic liver diseases. The current antiviral treatment for chronic hepatitis $B(C H B)$ is effective in controlling viral replication but fails to achieve a complete cure. Since the identification of sodium taurocholate cotransport polypeptide (NTCP) as an HBV receptor, anti-HBV drugs targeting viral entry, capsid assembly, cccDNA, transcription, and secretion have been developed. In this paper, the potential inhibitors in various steps of the HBV life cycle are summarized.

Key Words: Hepatitis B virus, Drugs, ccCDNA, ISG20

\section{INTRODUCTION}

Hepatitis B virus (HBV) is a partially $3.2 \mathrm{~kb}$ double-stranded DNA virus that infects hepatocytes. Persistent HBV infection is a major cause of chronic hepatitis $B$ $(\mathrm{CHB})$, cirrhosis, and hepatocellular carcinoma $(1,2)$. The natural history of HBV infection is divided into four phases according to serum alanine aminotransferase (ALT), serum HBV DNA levels, and HBeAg status (seroconversion): immune tolerant (IT), immune active or clearance (IA), inactive carrier (IC), and reactivation phase. Adults with acute HBV infection generally clear the infection spontaneously through the IT phase and the IA phase within $4-20$ weeks. However, 95\% of neonates, $2-30 \%$ of children, and less than $5 \%$ of adults develop CHB through the HBV reactivation phase (2). Currently, an estimated 250 million people are infected with HBV worldwide, resulting in 1.4 million deaths annually, and 10 30 million are newly infected. In particular, a half of the patients with $\mathrm{CHB}$ in the world are distributed throughout Asia, including China and India. In Korea, it is estimated that 2 to 3 million people are infected with HBV, and of whom 400,000 are CHB patients.

The FDA-approved antiviral agents administered to patients with CHB are modified interferon alpha (Peg-interferon) and nucleot(s)ide analogs. Peg-interferon has serious side effects including fever, chills, and heart failure, and shows less response to treatment because the IFN signal is blocked by HBV. Nucleot(s)ide analogs interfere with and terminate the DNA strands synthesized by HBV polymerase within the HBV capsid during the reverse transcription. Nevertheless, natural mutation by viral polymerase, which lacks proof-reading, occurs in the 
reverse transcriptase domain of polymerase, and consequently resists Lamivudine, Adefovir, Entecavir, and Tenofovir following long-term administration $(3,4)$. Therefore, it is impossible to completely cure CHB, suggesting that it is necessary to develop novel antiviral agents targeting the various steps of the HBV life cycle without drug resistance. In this review, potential drugs targeting viral entry, capsid assembly, cccDNA, transcription, and secretion are summarized.

\section{BODY}

\section{Life cycle of Hepatitis B virus}

HBV initially attaches to the heparin sulfate proteoglycans (HSPGs) on the cell surface, and then entry into hepatocytes through HBV large surface protein (Pre-S1) binding to sodium taurocholate cotransport polypeptide (NTCP) (5). After internalization of HBV, the capsid is released and moves into the nucleus, and then partially double-stranded viral DNA in the capsid is transformed to a covalently closed circular DNA (cccDNA). cccDNA, an episome type of mini-chromosome, is the template for subgenomic RNA (for surface and HBx protein) and major viral RNAs (pregenomic (pg) RNA and pre-core RNA that produces the viral antigen (HBeAg). Specifically, core protein and polymerase are translated by bicistronic pgRNA. pgRNA and polymerase is encapsidated into the capsid consist of core protein dimer. pgRNA in capsid works as the template for HBV rCDNA which synthesized by viral polymerase. Capsid reused for HBV rCDNA conversion to cccDNA in nucleus or bound to the surface protein on ER to form full HBV virions (Dane particles), and is then released $(1,6)$. Dane particles infect the surrounding liver cells or re-infect the cell to continuously proliferate HBV.

\section{Entry inhibitors}

Since the discovery of NTCP, an HBV receptor in 2012, many researchers have proposed an entry inhibitor. Myrcludex B, a lipopeptide derivative of the N-terminal of Pre-S1 and Cyclosporin A, a cyclic peptide binding to NTCP and its derivatives have been reported. It has also been reported that irbesartan and ezetimibe, which have been used as treatments on certain diseases, block HBV infection by suppressing NTCP (5).

\section{Myrcludex B}

Myrcludex B (MyrB, drug name; Bulevirtide) is a myristoylated peptide synthesized from 47 amino acids (a.a.2 - 48) at the $\mathrm{N}$-terminal of the PreS1 and binds to NTCP to interrupt the binding between HBV and NTCP, consequently inhibit the entry of HBV into hepatocytes (7). In addition, Myrcludex B inhibits HDV infection, which shares surface proteins with HBV. Combination therapy of Myrcludex $B$ with nucleot(s)ide analogs or interferon is effective in patients with chronic hepatitis $B$ and $D$ and that is currently under phase 2a clinical trial. However, no reduction effect of HBV surface protein (HBsAg) was observed in the case of monotherapy (8).

\section{Cyclosporin A}

Cyclosporin A (CSA) is known to be an immunosuppressant of the calcineurin (CN) inhibitors series used to prevent rejection after organ transplant, and to inhibit the transport activity of membrane transporters. It has been reported that CSA suppresses NTCP activity and inhibits the HBV entry using the small molecule screening system (9). However, the side effect have been reported that CSA inhibits bile acid transport of NTCP and increases the bile acid concentration in the blood, causing hypercholanemia and growth retardation. Recently, synthesized CSA derivatives (SYC) which do not affect the bile acid transport of NTCP have been developed (10). 


\section{Small molecules}

Recently, HBV entry inhibitors have been developed through drug repositioning. It has been reported that irbesartan, which is used as a treatment for hypertension, heart failure, and diabetic kidney disease, and ezetimibe, which is used as a treatment for hypertension and hyperlipidemia, inhibit HBV infection by inhibiting NTCP activity, but serious adverse effects such as hyperkalemia and hypotension were observed to develop due to their high blood concentration $(11,12)$.

\section{Capsid assembly inhibitor}

Capsid assembly inhibitors, which are divided into core protein allosteric modulators (CpAMs) and core assembly modulators (CAM) according to the mechanism of action, bind to HBV core proteins to interrupt the double-bond between proteins and then to inhibit capsid formation, or bind to the capsid to inhibit pgRNA encapsidation, HBV replication, and de novo cccDNA synthesis. CpAMs are small molecules of the heteroaryldihydropyrimidine (HAT) class that bind to the pocket between the core protein dimer-dimer and induce the formation of capsids with an abnormal structure, and they have been reported to be HAP1, HAP18, AT130, GLS4, and R07049389 (13, 14). CAMs are small molecules of the sulfamoylbenzamide class that induce the formation of capsids with an abnormal structure or an empty capsid formation lacking pgRNA, to prevent DNA replication and the full virion (virion progeny) formation of HBV $(14,15)$. Currently, there are JNJ6379 (phase 2 clinical trial), JNJ0440 (phase 1 clinical trial), and ABI-H0731 (phase 2 clinical trial); and NVR 3-778 was observed to have an HBV inhibitory effect for the first time in patients with chronic hepatitis B $(14,16)$. However, it is highly possible that resistance will develop due to spontaneous mutations of the core protein.

\section{cccDNA inhibitor}

HBV cccDNA exists as a mini-chromosome, which is surrounded by histone protein like the host cell's chromosome, in the nucleus of the host cell (6). All viral mRNAs are synthesized from cccDNA, and the expression of viral mRNA is regulated by posttranscriptional modifications (PTDs) such as methylation, acetylation, phosphorylation, and ubiquitination of the histone protein, as in the host cell. Hyper acetylation and methylation of histone open the cccDNA required for an active transcription process and HBV replication (17). Thus, methylation and acetylation inhibitors have been studied as drugs for inhibiting cccDNA transcription. Also, the CRISPR/Cas9 (CRISPR-associated system 9) method, which works directly on siRNA and ccCDNA, which inhibit RNA expression by targeting viral mRNA, has been developed.

\section{Epigenetic drugs (Epidrugs)}

Histone protein methylation and HBV DNA methylation have been reported to increase HBV replication, while FDA-approved methylation inhibitors (DNMT inhibitors), i.e. 5-azacytidine and 5-aza-2'-deoxycytidine, have been reported to inhibit HBV but with hepatotoxicity. Another methylation inhibitor, (-)-epigal-locatechin-3-gallate (EGCG), has been reported to inhibit HBV infection, but its effects on the chromosomes of the host cell are unknown, raising the need for further studies (17). It has been reported that the histone acetylation inhibitor (HAT inhibitor) C646 inhibits the acetylation of H3K27ac and H3K122ac and the transcription of ccCDNA in human primary hepatocyte without cytotoxicity (18).

\section{siRNA and CRISPR/Cas9}

siRNA, which directly targets mRNA (pgRNA, HBx) produced from HBV cccDNA, is currently under development as a drug, and VIR-2218 and RG6346 are currently undergoing phase 2 clinical trials. It has been reported that CRISPR/Cas9, which is designed to directly target ccCDNA, cuts cccDNA by working on the ORF of the HBV surface protein and the X protein (19), but such problems as intracellular delivery and cccDNA target specificity are yet to be resolved. 


\section{HBsAg secretion inhibitors}

Based on the fact that the HBV surface antibodies (HBsAg) in the blood neutralize the immune system of the host cell and promote HBV infection, nucleic acid polymers (NAPs) have been developed that inhibit the transport and secretion of HBV surface antibodies. Representative NAPs include REP compounds and ALG-10133. It has been confirmed that REP2139 and REP2165 inhibit the secretion of HBV surface antibodies in the cell and pre-clinical trial, and are currently under phase 2 clinical trial. Despite their proven effectiveness in the clinical trials, the exact nature of the inhibition mechanism remains unclear (20)

\section{Immune modulators}

To eliminate HBV completely or achieve a functional cure of the liver cells, it is necessary not only to develop materials that target various parts of the virus but also to restore the immune system of the host cell $(21,22)$. Accordingly, studies on toll-like receptors (TLR) and RIG-I agonists, which recognize HBV DNA or RNA and induce cytokine secretion to activate the innate immune system, are being performed: Inarigivir (RIG-I agonist, phase 2 clinical trial), RO7020531 (TLR7 agonist, phase 1 clinical trial), GS-9620 (TLR7 agonist, phase 2 clinical trial), and GS-9688 (TLR8 agonist, phase 1 clinical trial) are all undergoing clinical trials $(14,22)$, while the acquired immunity activating vaccines TG-1050 and HepTcell are currently undergoing phase 1 clinical trials (14).

\section{Recent study conducted by $\mathrm{KNIH}$ (Korea National Institute of Health): Interferon-stimulated gene 20 (ISG20)}

Interferons (IFNs) are main molecules of innate immune response against viral infection. ISG20 is a 3'-5' exonuclease and induced by type I and II IFNs, which mediated IFN-induced antiviral activity. However, anti-HBV activity and molecular mechanism of ISG20 on the HBV replication are poorly investigated. Recently, our study reported that ISG20 controlled HBV infection at the transcription level (23). The underlying mechanism of HBV inhibitory effect of ISG2O induced by IFN- $\beta$ is directly binding to Enhll/Cp region which contained the DNA binding sequence to transcriptional factor, consequently inhibited HBV transcription from viral enhancers. Finally, ISG20 expression was associated with natural history of HBV infection in patients with chronic hepatitis $B$. This findings revealed a novel mechanism of antiviral activity targeting various step of HBV life cycles.

\section{CONCLUSION}

To achieve the complete cure of CHB, it is necessary to develop drugs targeting the various step of HBV life cycle. Here, we introduced the several drugs targeting the HBV life cycle and immune modulators. Since these agents showed the less antiviral activity, side effect, and no effect of cccDNA reduction, the clinical trials in combination with nucleot(s)ide analog are under investigating. Although cytidine deaminase APOBEC3A, 3B induced by IFN- $\alpha$ and lymphotoxin- $\beta$ has been reported to degradate $c c c D N A$, its precise mechanism for $₫ \subset C D N A$ elimination has not yet been clearly demonstrated. Therefore, the underlying mechanism of cccDNA elimination should be investigated through the discovery of host factors such as tyrosyl-DNA-phophodiesterase (TDP2) involved in the conversion of rcDNA to cccDNA. 


\section{REFERENCES}

1) Seeger C, Mason WS. Molecular biology of hepatitis B virus infection. Virology 2015;672-86.

2) Trépo C, Chan HLY, Lok A. Hepatitis B virus infection. Lancet 2014;384:2053-63.

3) Ghany MG, Doo EC. Antiviral resistance and hepatitis B therapy. Hepatology 2009;49:S174-84.

4) Kim JH, Park YK, Park ES, Kim KH. Molecular diagnosis and treatment of drug-resistant HBV. World J Gastroentrol 2014;20:5708-20

5) Lempp FA, Urban S. Inhibitors of hepatitis B virus attachment and entry. Intervirology 2014;57:151-7.

6) Nassal M. HBV cccDNA: viral persistance reservoir and key obstacle for a cure of chronic hepatitis B. Gut 2015:64:1972-84.

7) Gripon P, Cannie I, Urban S. Efficient inhibition of hepatitis B virus infection by acylated peptides derived from the large viral surface protein. J Viro/2005;79:1613-22.

8) Spyrou E, Smith Cl, Ghany MG. Hepatitis B: Current Status of Therapy and Future Theapies. Gastroenterol Clin North Am 2020;49:215-38.

9) Watashi K, Sluder A, Daito T, Matsunaga S, Ryo A, Nagamori S, et al. Cyclosporine A and its analogs inhibit hepatitis $B$ virus entry into cultured hepatocytes therough targeting a membrane transporter, sodium taurocholate cotransporting polypeptide (NTCP). Hepatology 2014;59:1726-37.

10) Shimura S, Watashi K, Fukano K, Peel M, Sluder A, Kawai F, et al. Cyclosporin derivatives inhibits hepatitis B virus entry without interfering with NTCP transporter activity. J Hepato/2017:66:685-92.

11) Wang XJ, Hu W, Zhang TY, Mao YY, Liu NN, Wang SQ. Irbesartan, an FDA approved drug for hypertension and diabetic nephropathy, is a potent inhibitor for hepatitis $\mathrm{B}$ virus entry by disturbing $\mathrm{Na}(+)$-dependent taurocholate cotransporting polypeptide activity. Antiviral Res 2015:120:140-6.

12) Lucifora J, Esser K, Protzer U. Ezetimibe blocks hepatitis $B$ virus infection after virus uptake into hepatocytes. Antiviral Res 2013:97:195-7.

13) Venkatakrishnan B, Katen SP, Francis S, Chirapu S, Finn MG, Zlotnick A. Hepatitis B virus capsids have diverse structural responses to small-molecule ligands bound to the heteroaryldihydropyrimidine pocket. J Virol 2016:90:3994-4004.

14) Fanning GC, Zoulim F, Hou J, Bertoletti A. Therapeutic strategies for hepatitis B virus infection : towards a cure. Nat Rev Drug Discov 2019;18:827-44.

15) Lam AM, Ren S, Espiritu C, Kelly M, Lau V, Zheng L, et al. Hepatitis B virus capsid assembly modulators, but not nucleoside analogs, inhibit the production of extracellular pregenomic RNA and spliced RNA varients. Antimicrob Agents Chemother 2017:61:e00680-17.

16) Lam AM, Espiritu C, Vogel R, Ren S, Lau V, Kelly M, et al. Preclinical Characterization of NVR 3-778, a First-in-Class Capsid Assembly Modulator against Hepatitis B Virus. Antimicrob Agents Chemother 2018;63;e01734-18.

17) Hong $X$, Kim ES, Guo H. Epigenetic regulation of hepatitis B virus covalently closed circular DNA: implications for epigenetic therapy against chronic hepatitis B. Hepatology 2017:66:2066-77. 
18) Tropberger P, Mercier A, Robinson M, Zhong W, Ganem DE, Holdort M. Mapping of histone modifications in episomal HBV cccDNA uncovers an unusual chromatin organization amenable to epigenetic manipulation. Proc Natl Acad Sci U S A 2015;112:E5715-24.

19) Karimova M, Beschomer N, Dammermann W, Chemnitz J, Indenbirken D, Bockmann JH, et al. CRISPR/Cas9 nickase-mediated disruption of hepatitis B virus open reading frame S and X. Sci Rep 2015:5:13734.

20) Durantel D, Zoulim F. New antiviral targets for innonvative treatment concepts for hepatitis B virus and hepatitis delta virus. J Hepato/ 2016:64:S117-31.

21) Levrero M, Testoni B, Zoulim F. HBV cure: why, how, when?. Curr Opin Viro/2016;18:135-43.

22) Sato S, Li K, Kameyama T, Hayashi T, Ishida Y, Murakami S, et al. The RNA sensor RIG-I dually functions as an innate sensor and direct antiviral factor for hepatitis B virus. Immunity 2015;42:123-32.

23) Park YK, Lee SY, Lee AR, Kim KC, Kim K, Kim KH, et al. Antiviral activity of interferon-stimulated gene 20, as a putative repressor binding to hepatitis B virus enhancer II and core promoter. J Gastroenterol hepato/2020;35:1426-36. 